\title{
Energy Absorption and Crushing Behaviour of Foam-Filled Aluminium Tubes
}

\author{
Yasuo Yamada ${ }^{1}$, Takumi Banno ${ }^{1}$, Zhenkai Xie $^{1}$ and Cui'e Wen ${ }^{2}$ \\ ${ }^{1}$ Materials Research Institute for Sustainable Development, National Institute of Advanced Industrial Science and Technology (AIST), \\ Nagoya 463-8560, Japan \\ ${ }^{2}$ Faculty of Science \& Technology, Deakin University, Geelong, VIC 3217, Australia
}

\begin{abstract}
The crushing behaviour and energy absorption of foam filled aluminium tubular structures were investigated using the quasi-static compressive tests. The crushing behaviour of the tubular structures changed due to foam filling. The energy absorption of the foam filled tubular structures was improved significantly. Foam filling caused an interaction effect between the tube and the foam during progressive crushing, leading to an increase in the mean crushing load compared to that of the foam or tube itself. This interaction effect might be affected by several parameters such as the density of the foam, the properties of both the foam material and tube material, and the thickness and outer diameter of the tube. In particular, the interaction effect essentially depended on the ratio of the mean crushing force of the foam to that of the tube.
\end{abstract}

(Received June 20, 2005; Accepted August 12, 2005; Published December 15, 2005)

Keywords: porous materials, aluminium foam, foam-filled metal tubes, concertina mode, diamond mode, interaction effect

\section{Introduction}

The reduction of vehicle mass is receiving increasing attention in both the eco-society and auto-manufacturers due to global warming and environmental pollution. However, the combined requirements to vehicle manufacturers to improve crashworthiness and comfort; and provide features such as air conditioning and power steering have led to increased weights of new vehicle models. Change of design geometry and application of new eco-materials are the two basic approaches that are now being considered to satisfy the mass, comfort and safety requirements.

Metal foams exhibit unique combinations of physical, mechanical, thermal, electrical and acoustic properties. ${ }^{1,2)}$ In particular, they are light and good at absorbing energy. Such superior characteristics make them new attractive ecomaterials in automotive applications where crashworthiness and weight are critical. Aluminium foams are of particular practical interest due to the recent developments of costeffective production processes. ${ }^{3,4)}$ The long plateau behaviour of the stress-strain curves of aluminium foams make them ideal materials for energy absorption.

The deformation behaviour of thin-walled metallic tubes subjected to axial compression has been studied for many years. ${ }^{5,6)}$ Such tubes have been known as excellent impact energy absorbers because of their progressive axial folding. Recently, there has been an increasing interest in using aluminium foams as cores inside the thin-walled metallic tubes for energy absorption. ${ }^{7-9)}$ Metallic tubes filled with aluminium foam cores are attractive because of the interaction between the tube wall and the foam core. ${ }^{10-12)}$ The crushing forces of foam-filled tubes are higher than the algebraic sum of the crushing forces of tube or foam itself, resulting from the interaction effect. Thus the energy absorption of the foam-filled tubes is greater than that of the component tube and foam. However, there is still insufficient data to understand the mechanism of the interaction between the tube wall and the foam core. In the present study, the compressive tests were carried out on circular aluminium tubes and tubes filled with aluminium foams. The intention was to study the mechanism of interaction effect between the tube and foam. X-ray Computed Tomography (X-CT) was employed to image the internal structure of foam samples during straining nondestructively.

\section{Experimental Procedures}

Circular tubes used in this research were made up of aluminium alloy A6063 (Si 0.2-0.6, Mg 0.45-0.9, Fe 0.35, $\mathrm{Cu}<0.1)$. All tubes were initially delivered in a $\mathrm{T} 5$ condition and further annealed at $450^{\circ} \mathrm{C}$ for $2 \mathrm{~h}$ in the present study. The length/outer diameter ratio $(L / D)$ and the wall thickness/outer diameter ratio $(t / D)$ of the tubes were selected to obtain two kinds of deformation modes, i.e. concertina mode and diamond mode. ${ }^{13)}$ To study the interaction effect between the tube and the foam, tubes with various wall thickness/outer diameter ratios $(t / D)$ were prepared. Dimension parameters and properties of tubes were listed in Table 1.

Aluminium foam samples with porosities ranging from 89 to $92 \%$ and a closed-cell structure (trade name Alporas, Shinko Wire Co. Ltd., Japan) were used as foam core in the present study. The aluminium foam was produced by adding calcium and titanium hydride powder into melted aluminium alloy. The calcium acted to increase the viscosity

Table 1 Dimension parameters and properties of aluminium tubes.

\begin{tabular}{ccccc}
\hline Tube & $\begin{array}{c}\text { Outer diameter } \\
(D / \mathrm{mm})\end{array}$ & $\begin{array}{c}\text { Wall thickness } \\
(t / \mathrm{mm})\end{array}$ & $\begin{array}{c}\text { Length } \\
(L / \mathrm{mm})\end{array}$ & $\begin{array}{c}\sigma_{0.2} \text { (Annealed tubes) } \\
(\mathrm{MPa})\end{array}$ \\
\hline Group A & 22 & 2.0 & 77 & 105 \\
& 22 & 1.5 & 77 & 105 \\
& 22 & 1.0 & 77 & 105 \\
\hline \multirow{2}{*}{ Group B } & 40 & 1.0 & 100 & 105 \\
& 50 & 1.0 & 100 & 105 \\
& 76 & 1.5 & 160 & 105 \\
& 76 & 1.0 & 160 & 105 \\
\hline Group C & 76 & 0.5 & 160 & 105 \\
\hline
\end{tabular}



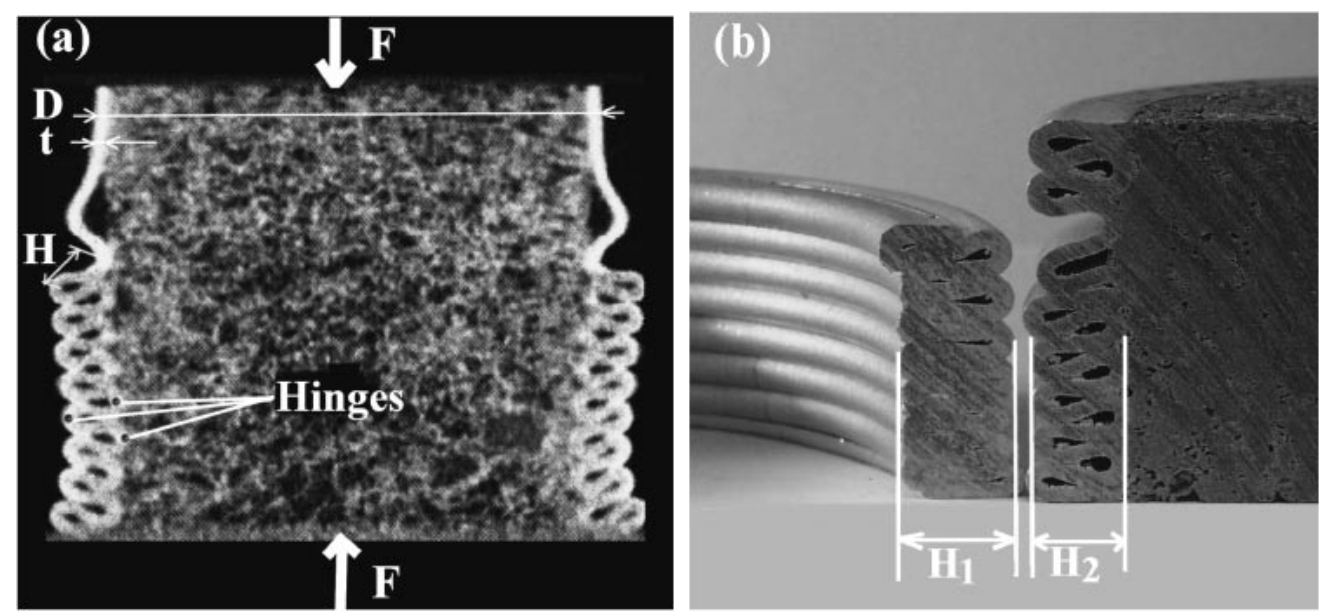

Fig. 1 (a) X-CT photograph of an interrupted compressive process; (b) cross-section photographs of an empty tube and a foam-filled tube after compression, showing a decrease in the buckle hinge length $\left(H_{2}<H_{1}\right)$.

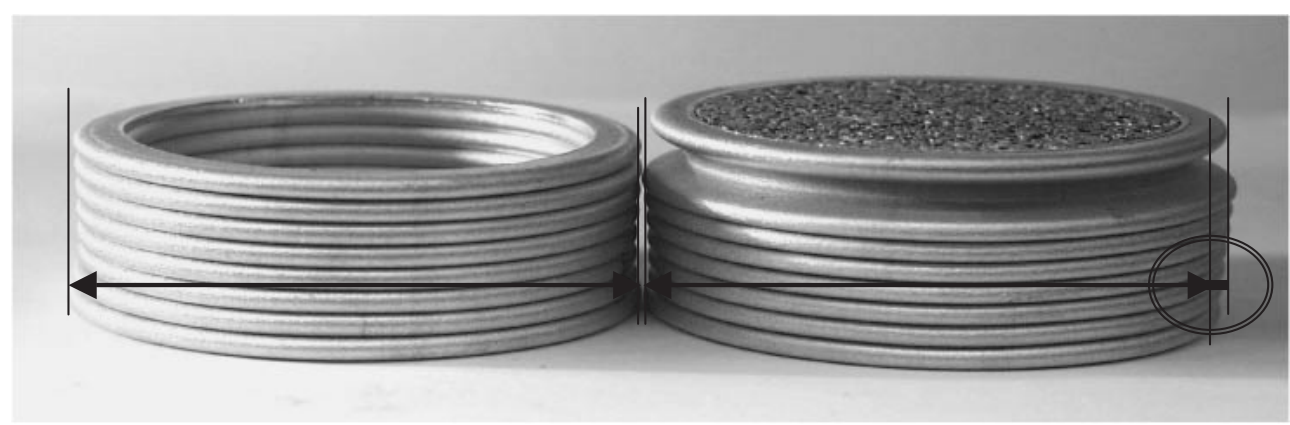

Fig. 2 Photographs of the axially crushed empty tube and the foam filled tube, showing the same crushing mode of concertina; but the progressive buckling expanded outwards as marked in the circle.

of the molten, while the titanium hydride decomposed and therefore released gaseous hydrogen, which formed bubbles in the molten. ${ }^{14)}$ The chemical composition of the foam was $\mathrm{Al}-1.42 \mathrm{Ca}-1.42 \mathrm{Ti}-0.28 \mathrm{Fe}-0.007 \mathrm{Mg}$ (mass $\%$ ). The foam material was supplied in a large panel with the dimensions of $1500 \mathrm{~mm} \times 750 \mathrm{~mm} \times 450 \mathrm{~mm}$. The average cell diameter of the aluminium foam sample was $4.0 \mathrm{~mm}$. The density of each aluminium foam sample was determined by measuring its weight and volume. Foam samples with diameters and lengths that fit their tube counterparts were cut from the centre part of the large panel. The diameters of the foam cores were machined to be very close to the inner diameters of the tubes so that the foam core fitted tightly inside the tubes.

In order to determine the deformation behaviour and the energy absorption of a sample, compressive tests were carried out to crush the tube samples axially and the forcedisplacement relationships were recorded. The mean crushing force and the Specific Energy Absorption (SEAenergy absorbed per unit mass $[\mathrm{kJ} / \mathrm{kg}]$ ) were then calculated. Compressive tests were carried out at a fixed strain rate of $1 \times 10^{-3} \mathrm{~s}^{-1}$ at room temperature. To characterize the deformation behaviour, X-ray computed tomography was employed to image the internal structures of tubes filled with foam cores after various periods of strains nondestructively.

\section{Results and Discussion}

The deformation behaviour of group $A$ and group $B$ empty tubes showed the typical progressive axisymmetric crushing via the concertina mode. Group $C$ empty tube deformed in a progressive non-axisymmetric manner via the diamond mode. Foam filling did not change the deformation mode for the group $A$ and group $B$ tubes; but it caused a decrease in the buckle hinge length as shown in Fig. 1. Figure 1(a) indicates the outer diameter $(D)$, the wall thickness $(t)$, and the buckling hinges length $(H)$ of a foam-filled tube during progressive crushing via a concertina mode. Figure 1(b) shows the decrease in the buckle hinge length of a tube by foam filling. This tube sample has the parameters of an outer diameter $76 \mathrm{~mm}$, wall thickness $1.5 \mathrm{~mm}$ and length $160 \mathrm{~mm}$; the porosity of the foam core is $90 \%$. The buckle hinge length decreased $1.6 \mathrm{~mm}$ due to foam filling. The decrease in the buckle hinge length depended on the ratio of wall thickness to outer diameter ratio $(t / D)$ and the properties of the tube and the foam. Observations found that the decrease in the buckle hinge length is larger for group $B$ foam-filled tubes than group $A$ ones.

Moreover, foam filling also caused the progressive buckling expanding outwards noticeably compared to the nonfilled tubes. Figure 2 shows the photographs of an axially crushed empty tube and a foam filled tube, demonstrating the 


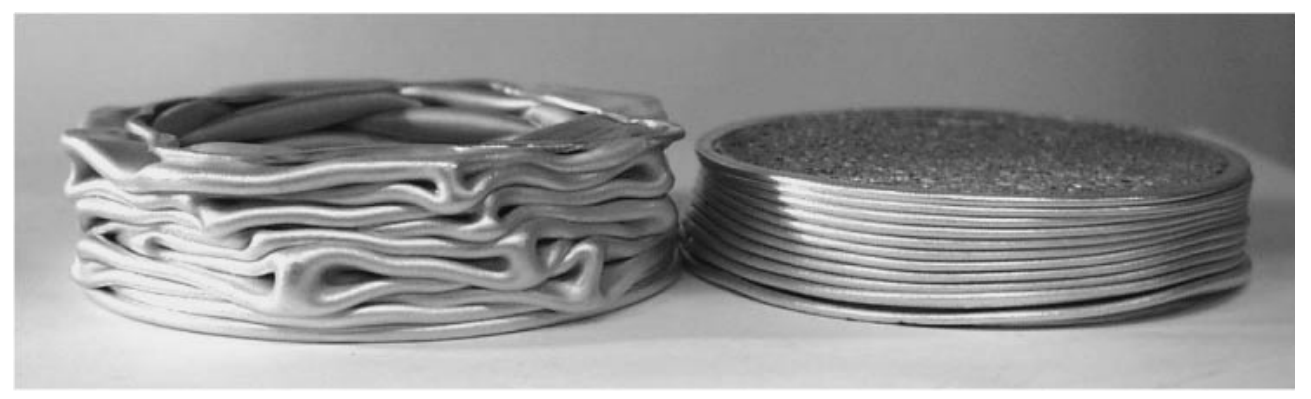

Fig. 3 Photographs of the axially crushed empty tube and the foam filled tube, showing that progressive crushing mode changed from non-axisymmetric diamond mode to axisymmetric concertina mode.

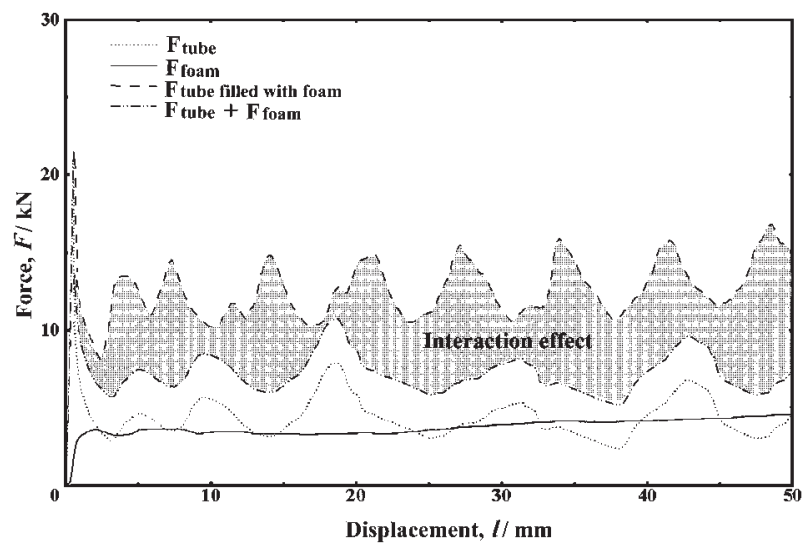

Fig. 4 Illustration of the interaction effect between the tube and foam.

same progressive crushing mode of concertina but an outwards expansion of the progressive buckling. The tubes were of the same parameters as an outer diameter $50 \mathrm{~mm}$, length $100 \mathrm{~mm}$ and wall thickness $1 \mathrm{~mm}$. The porosity of the foam core was $90 \%$. The outwards expansion in outer diameter was $1.5 \mathrm{~mm}$. It should be noted that the outwards expanding is larger for group $B$ foam-filled tubes than group $A$ ones, which is in consistent with the phenomenon of the decrease in the buckle hinge length.

In group $C$, however, the foam filling changed the deformation mode from diamond mode to concertina mode. Figure 3 shows the photographs of an axially crushed empty tube and a foam filled tube, demonstrating the deformation mode shift from diamond mode to concertina mode due to foam filling. The empty tube had an outer diameter $76 \mathrm{~mm}$, wall thickness $0.5 \mathrm{~mm}$ and length $160 \mathrm{~mm}$. The porosity of the foam core was $92 \%$.

For all the foam filled tubes, there was an interaction effect between the tube and foam, directly reflected in the compressive force-displacement curves. The crushing forces of the foam-filled tubes were greater than the sum of the crushing force of tube or the foam itself. The interaction effects of group $C$ samples were larger than that of the group $B$ and group $A$ samples. The typical interaction effect of foam filling on the force-displacement curve is plotted in Fig. 4, where the outer diameter of the tube was $76 \mathrm{~mm}$, the wall thickness $0.5 \mathrm{~mm}$, and the porosity of the foam was $92 \%$. The interaction effect between the tube and foam caused an improvement in the SEA up to $75 \%$.

$\mathrm{X}$-ray computed tomography observations indicated that the deformation behaviour of an aluminium foam inside of a tube was distinctly different from that of a foam itself. The deformation of an aluminium foam sample without the outside tube under compressive loading was localised in narrow continuous deformation bands having widths of order of a cell diameter. Cell shapes affected the deformation behaviour significantly. Those cells with shape irregularities and defects such as corrugations, curvatures, and nonuniformities in the cell wall thickness that collapsed initiated the deformation bands in the foam sample. On the other hand, the deformation behaviour of an aluminium foam inside of a tube was restricted by the outside tube. Figure 5 shows that
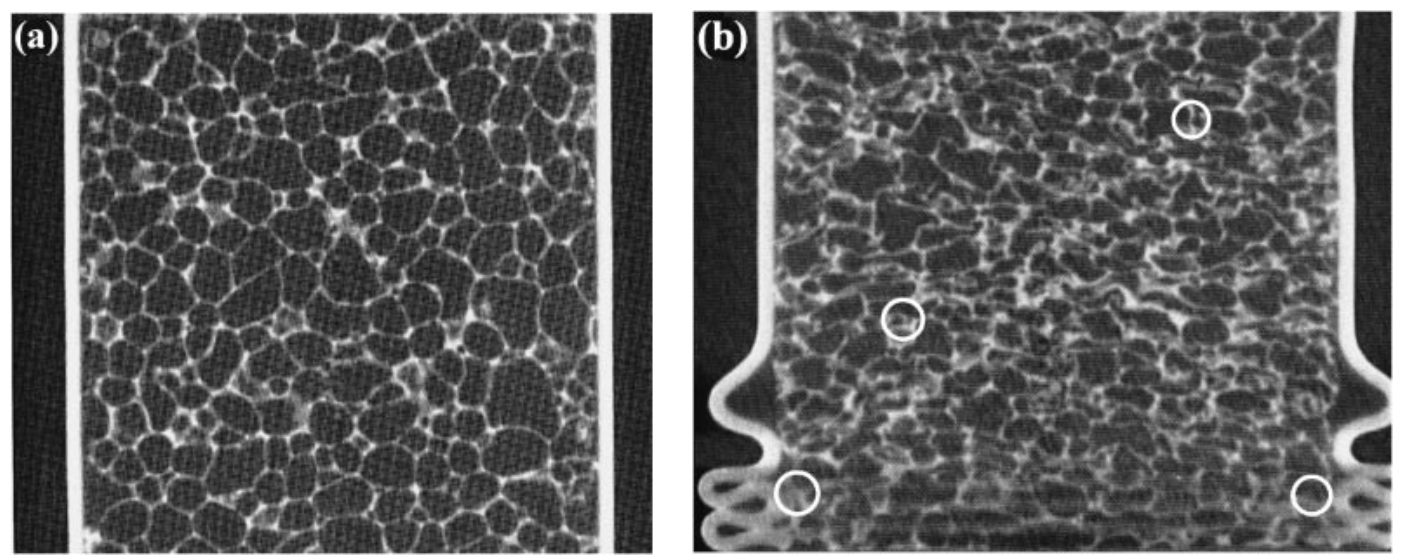

Fig. 5 X-CT photographs of a foam filled tube before crushing (a); and the interrupted axisymmetric buckling process of the foam filled tube; circles marked the deformation-band initiations (b). 


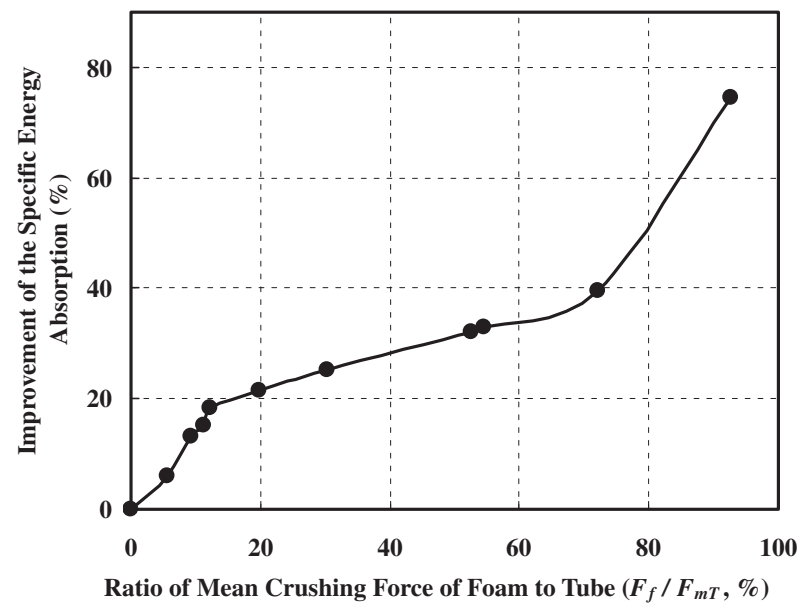

Fig. 6 Correlation between the improvement of SEA and the ratio of mean crushing force of foam to that of tube.

the deformation bands initiated in the areas contacted with the inwards buckling hinge of the tube, as well as those initiated in the areas with irregular and defective cells. This deformation preference obviously contributed to the interaction effect between the tube and foam. This selective deformation behaviour of metal foams provided a resistance to the inwards deformation of the tube wall; hence it caused an outwards expanding of the progressive buckling of tube wall, or a shift of deformation mode from diamond to concertina.

For all the crushed samples, the energy absorption was improved by the foam filling. The improvement in SEA, i.e. the interaction effect between the tube and foam, might be affected by the tube dimension parameters and the properties of the foam and the tube. However, the improvement in SEA essentially depended on the ratio of the mean crushing force of foam to that of the tube. Figure 6 shows the correlation between the improvement of SEA and the ratio of mean crushing forces. In the case of the sample with a mean crushing force ratio of 0.93 , the improvement in SEA reached $75 \%$. This corresponded to the deformation mode shift from diamond to concertina.

\section{Conclusions}

The present study investigated the crushing behaviour and energy absorption of the foam-cored aluminium tubular structures using compressive tests. X-ray computed tomography observations indicated that the deformation bands initiated in the areas contacted with the inwards buckling hinge of the tube, as well as those initiated in the areas with irregular and defective cells. This selective deformation behaviour of metal foams contributed to the interaction effect between the tube and foam. The energy absorption of the foam-cored structures was improved significantly. The interaction effect between the tube and the foam essentially depended on the ratio of the mean crushing force of foam to that of the tube.

\section{REFERENCES}

1) L. J. Gibson and M. F. Ashby: Cellular Solids, Structure and Properties, (2nd edn., Cambridge University Press, Cambridge, 1997) pp. 1-510.

2) J. Banhart: Prog. Mater. Sci. 46 (2001) 559-632.

3) G. J. Davis and S. Zhen: J. Mater. Sci. 18 (1983) 1899-1911.

4) J. Baumeister, J. Banhart and M. Weber: Powder Metall. Int. 25 (1993) 182-185.

5) N. Jones: Structural Impact, (Paperback Edition, Cambridge University Press, 1997) pp. 1-537.

6) J. M. Alexander: Q. J. Mech. Appl. Math. 13 (1960) 11-16.

7) S. R. Reid, T. Y. Reddy and M. D. Gray: Int. J. Mech. Sci. 28 (1986) 295-322.

8) T. Y. Reddy and R. J. Hall: Int. J. Impact Eng. 7 (1998) 151-176.

9) W. Abramowicz and T. Wierzbicki: Int. J. Mech. Sci. 30 (1988) 263271.

10) A. G. Hanssen, M. Langseth and O. S. Hopperstad: Int. J. Impact Eng. 24 (2000) 475-507.

11) W. Chen and T. Wierzbicki: Thin-Walled Struct. 39 (2001) 287-306.

12) M. Seitzberger, F. G. Rammerstorfer, R. Gradinger, H. P. Degischer, M. Blaimschein and C. Walch: Int. J. Solid. Struct. 37 (2000) 41254147.

13) K. R. F. Andrews, G. L. England and E. Ghani: Int. J. Mech. Sci. 35 (1983) 687-696.

14) T. Miyoshi, M. Itoh, S. Akiyama and A. Kitahara: Adv. Eng. Mater. 2 (2000) 179-183. 